\title{
Analysis of Amplify-and-Forward Cooperative Networks with Nakagami-m fading Channels for MRC Diversity Combining
}

\author{
Ali Abdulwahhab Mohammed ${ }^{*^{1,2}}$, Li Yu ${ }^{1}$, Manar Al-Kali ${ }^{1}$, Desheng Wang ${ }^{1}$ \\ ${ }^{1}$ Division of Communication \& Intelligent Networks, Wuhan National Laboratory for Optoelectronics, \\ Huazhong University of Science \&Technology, Wuhan 430074, China \\ ${ }^{2}$ Department of Electrical Engineering, Faculty of Engineering, University of Baghdad, Baghdad, Iraq \\ ${ }^{*}$ Corresponding author, e-mail: alialhashmi1968@yahoo.com
}

\begin{abstract}
In this paper we study the effect of diversity combining for Nakagami -m fading Channels in the amplify-and-forward cooperative networks. We derive the cumulative density function (CDF), probability density function (PDF) and moment generating function (MGF) for the multiple relay amplify -and-forward network with single half duplex channel maximal ratio combiner (MRC). In this network we investigate the cases of MRC at the destination, as well derive the exact Symbol Error Rate (SER) of M-ary phase-shift keying (M-PSK), and quadrature amplitude modulation (M-QAM) in Nakagami - $m$ fading environment. We present a comparison between M-PSK and M-QAM modulation schemes in some representative scenarios where an arbitrary number of cooperative relays is considered.
\end{abstract}

Keywords: cooperative networks, amplify-and-forward, maximal ratio combining, nakagami $-m$ fading channels

Copyright $₫ 2015$ Institute of Advanced Engineering and Science. All rights reserved.

\section{Introduction}

It is well known that the concept of cooperative networks relay recently developed a keen interest in the research community due to its ability to improve the reliability of data transfer and expand the coverage of wireless networks [1-2].

Nakagami-m fading distribution covers a wide range of fading scenarios via the $m$ $(m>0.5)$ parameter, which includes the Rayleigh $m=1$ as a special case [3].

In [4-5], the authors investigate the impact of multiple-antenna destination reception in the performance of AF cooperative diversity systems that was investigated in Rayleigh fading environment.However, the symbol error probability (SEP) analysisis limited to the case when the source-to-destination $(S \rightarrow D)$ and relay-to-destination $(R \rightarrow D)$ links are balanced with equal average signal-to-noise ratio (SNR), and the SNR of the $S \rightarrow R$ link is sufficiently larger than that of the $S \rightarrow D$ Link.

The authors in [6-7] shows the symbol error probability (SEP) of BPSK and M-ray performance maximal ratio combining (MRC) diversity operating in Nakagami-m and Rayleigh fading Wireless channels.

In [8-9] the error performance of the MRC with transmits antenna selection and receiver maximal-ratio combining (TAS/MRC) scheme is investigated in independent flat Nakagami-m fading channels for arbitrary m.Is attained from two differentapproaches. Then the exact symbol error rate (SER) expressions are attained as a multiple infinite sum based on the moment generating function (MGF) method for M-ary phase-shift keying (M-PSK) and M-ary quadrature amplitude modulation (M-QAM) [10]. The asymptotic SER expressions reveal a diversity order equal to the product of the $\mathrm{m}$ parameter.The authors in [11] derived a conditional lower bound for the error rate and the outage probability (OP) of the cooperative diversity networks over independent and non-identical Nakagami-m fading channels with AF relaying and maximum ratio combining (MRC) at the destination node.

This paper presents the analysis of AF cooperative network in Nakagami-m fading environment with MRC and $\mathrm{N}$ single antenna relays. We also provide analyzing and deriving the 
exact sample error rate (SER) performance of maximal ratio combining receivers over Nakagami-m fading channels based on the derived PDF, CDF and MGF of the out dual channel MRC. The novel obtained expression is then used to Study the effect of diversity reception error on the performance of the MRC with M-ary phase-shift keying (M-PSK) and M-ary quadrature amplitude modulation (M-QAM) with different values of the ratio of the decision after a quadrature phase over Nakagami -m Fading Channels.

The remainder of this paper is organized as follows: Section 2 describes the system model. We derive the PDF, CDF and MGF of the out dual channel maximal ratio combiner (MRC) in Section 3 Derivation SER with different modulation in Section 4 numerical results in Section 5 conclusions.

\section{System Model}

we describe in this section the cooperative network distributed the contract Source $S$ communicates with the destination node $D$ with the help of ith relay nodes, $\left(R_{1}, R_{2}, \ldots \ldots ., R_{i}\right)$, using amplify - and - forward protocol as shown in Figure 1.

Each node is equipped with an antenna in the independent flat Nakagami-m fading channels and operates in half - duplex mode. The signals are transmitting as an orthogonal presumably either in time or frequency. The corresponding fading coefficients for the $S \rightarrow D$, $S \rightarrow R_{i}$ and $R_{i} \rightarrow D$ are $h_{S D}, h_{S R_{i}}$ and $h_{R_{i} D}$, are fading channel respectively. Due to Nakagami $\mathrm{m}$ fading Channels.

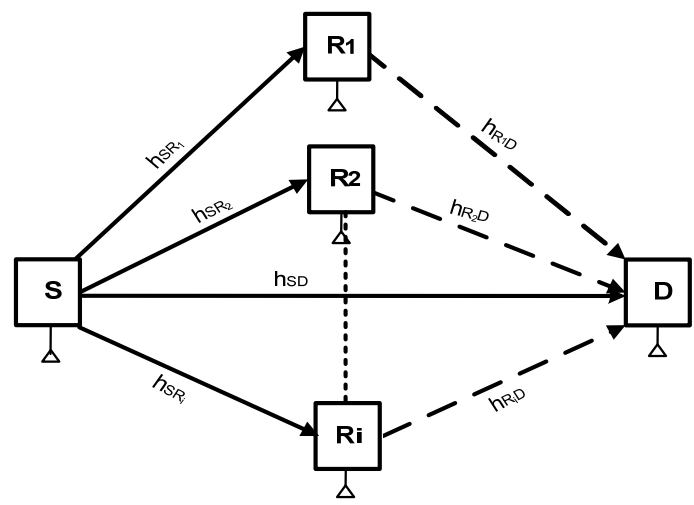

Figure 1. System model Cooperative network with $\mathrm{S}, \mathrm{n}$ relays and $\mathrm{D}$

The signals received at the destination and the $i^{\text {th }}$ relay, the system model can be described by the following set of equation as follows:

$$
\begin{aligned}
& y_{S R_{i}}=h_{S R_{i}} \sqrt{P_{s}} x+\eta_{S R_{i}} \\
& y_{S D}=h_{S D} \sqrt{P_{s}} x+\eta_{S D}
\end{aligned}
$$

$x$ is the transmitted signal, $P_{S}$ is transmitted source power, $\eta_{S D}$ and $\eta_{S R_{i}}$ as well as $\eta_{R_{i} D}$ are all additive White Gaussian noises (AWGN) with variances No .

$$
y_{R D_{i}}=h_{R D_{i}} \sqrt{P_{r}} x_{r}+\eta_{R_{i} D}
$$


Where $x_{r}=G y_{S R_{i}}, \mathrm{G}$ is the amplified factor, $P_{r}$ is the received power at the $\mathrm{i}^{\text {th }}$ relay, and then $x_{r}$ is the information on the relay. Using MRC we get:

$$
G=\sqrt{\frac{P_{r}}{\left|h_{S R_{i}}\right|^{2} P_{S}+N_{O}}}
$$

From (3) the instantaneous end-to-end SNR can be expressed as follows:

$$
\gamma_{i}=\frac{\gamma_{S R_{i}} \gamma_{R_{i} D}}{\gamma_{S R_{i}}+\gamma_{R_{i} D}+1}
$$

Let us define the effective instantaneous SNRs for source to destination, source to the $i^{\text {th }}$ relay and $i^{\text {th }}$ relay to destination links as $\gamma_{S R_{i}}=\left|h_{S R_{i}}\right|^{2} P_{S} / N_{o}, \gamma_{R_{i} D}=\left|h_{R_{i} D}\right|^{2} P_{r} / N_{o}$ and $\gamma_{S D}=\left|h_{S D}\right|^{2} P_{S} / N_{o}$ respectively. For the direct link. The CDF of $\gamma_{d}$ can be expressed as in [12]:

$$
F_{\gamma_{d}}(\gamma)=\frac{\Omega\left(m, \bar{\gamma}_{d}\right)}{\Gamma\left(m_{i}\right)}
$$

Where $\Omega$ (.), $\Gamma$ (.) are the incomplete Gamma functions and $m_{i}$ is the Nakagami-m Fading parameter. Then the PDF can be expressed as follow:

$$
f_{\gamma_{i}}(\gamma)=\frac{\bar{\gamma}_{k}}{\Gamma\left(m_{i}\right)} \gamma^{m_{i}} e^{\left(-\gamma / \overline{\gamma_{i}}\right)}
$$

Then the PDF of $\gamma_{i}$ can be expressed as in (7).

$$
f_{\gamma_{i}}(\gamma)=\sum_{\substack{i=1 \\ j}}^{N} \prod_{\substack{j=1 \\ j \neq i}}^{N} P_{i} P_{j} \Omega\left(m_{j}, \bar{\gamma}_{j}\right) \gamma^{m_{i}} e^{\left(-\overline{\gamma_{i}}\right)}
$$

Where $P_{i}=\frac{\bar{\gamma}_{i}}{\Gamma\left(m_{i}\right)}, P_{j}=\frac{1}{\Gamma\left(m_{j}\right)}$

The CDF of the Cooperative path can be expressed by integrating its PDF in (8) as follows:

$$
\begin{aligned}
& F_{\gamma_{i}}(\gamma)=\int_{0}^{\gamma} f_{\gamma_{i}}(x) d x \\
& F_{\gamma_{i}}(\gamma)=\int_{0}^{\gamma} \sum_{i=1}^{N} \prod_{j=1}^{N} P_{i} P_{j} \Omega\left(m_{j}, \bar{\gamma}_{j}\right) x^{m_{i}} e^{\left(-x \bar{\gamma}_{i}\right)} d x \\
& F_{\gamma_{i}}(\gamma)=\sum_{i=1}^{N} \prod_{\substack{j=1 \\
j \neq i}}^{N} P_{i} P_{j} \frac{\left(\bar{\gamma}_{j}\right)^{m j} \Gamma\left(m_{i}+m_{j}-1\right)}{m_{j}\left(\bar{\gamma}_{j}+\bar{\gamma}_{i}\right)^{\left(m_{i}+m_{j}\right)}} \times{ }_{2} F_{1}\left(1, \alpha_{1}, \alpha_{2}, \alpha_{3}\right)
\end{aligned}
$$

Where $\alpha_{1}=m_{i}+m_{j}, \alpha_{2}=m_{j}+1, \alpha_{3}=\frac{\bar{\gamma}_{j}}{\bar{\gamma}_{j}+\bar{\gamma}_{i}}$

TELKOMNIKA Vol. 16, No. 3, December 2015: 546 - 552 
The corresponding MGF is given by:

$$
M_{g}(\gamma)=\int_{0}^{\infty} e^{-s t} \cdot f(t) \cdot \mathrm{dt}
$$

Using the identity in [13.eq(6.455.2)], we get the MGF of MRC receiver:

$$
M_{g i}(\gamma)=\sum_{i=1}^{N} \prod_{j=1}^{N} P_{j \neq i} P_{j} \frac{\left(\bar{\gamma}_{j}\right)^{m j} \Gamma\left(m_{i}+m_{j}-1\right)}{m_{j}\left(\bar{\gamma}_{j}+\bar{\gamma}_{i}+s\right)^{\left(m_{i}+m_{j}\right)}} \times{ }_{2} F_{1}\left(1, \alpha_{1}, \alpha_{2}, \alpha_{3}\right)
$$

Where $\alpha_{1}=m_{i}+m_{j}, \alpha_{2}=m_{j}+1, \alpha_{3}=\frac{\bar{\gamma}_{j}}{\bar{\gamma}_{j}+\bar{\gamma}_{i}}$

\section{Derivations SER, Performance of MRC}

In this section, we derive the SER of M-PSK and M-QAM modulations over Nakagami$\mathrm{m}$ fading channel.

\subsection{M-PSK Modulation}

The average SER for M-PSK signals can be written as [14].

$$
P_{S E R}=\frac{1}{\pi} \int_{0}^{\frac{(M-1) \pi}{M}} M_{g i}\left(\frac{g_{M-P S K}}{\sin ^{2} \theta}\right) d \theta
$$

Where $g_{M-P S K}=\sin ^{2}(\pi / M)$

$$
P_{S E R}=\sum_{i=1}^{N} \prod_{\substack{j=1 \\ j \neq i}}^{N} P_{i} P_{j} \int_{0}^{\frac{\pi(m-1)}{m}} \frac{\left(\bar{\gamma}_{j}\right)^{m j} \Gamma\left(m_{i}+m_{j}-1\right)}{m_{j}\left(\bar{\gamma}_{j}+\bar{\gamma}_{i}+s\right)^{\left(m_{i}+m_{j}\right)}} \mathrm{d} \theta \times{ }_{2} F_{1}\left(1, \alpha_{1}, \alpha_{2}, \alpha_{3}\right)
$$

Using the Binomial Theorem with the help of [13.eq(3.621.4)] we get:

$$
\begin{array}{r}
P_{S E R}=\sum_{i=1}^{N} \prod_{j=1}^{N} P_{i} P_{j} \times\left(\bar{\gamma}_{j}\right)^{m j} \Gamma\left(m_{i}+m_{j}-1\right) \frac{1}{m_{j}} \times \sum_{t=0}^{w}\left(\bar{\gamma}_{j}+\bar{\gamma}_{i}\right)^{-w+t} \\
\times{ }_{2} F_{1}\left(1, \alpha_{1}, \alpha_{2}, \alpha_{3}\right) \int_{0}^{\frac{\pi(m-1)}{m}}\left(\frac{g_{p s k}}{\sin ^{2} \theta}\right)^{-t} \cdot \mathrm{d} \theta \\
P_{S E R}=\sum_{\substack{i=1 \\
j=1} \prod_{j \neq i}^{N} P_{i} P_{j}} \times\left(\bar{\gamma}_{j}\right)^{m j} \Gamma\left(m_{i}+m_{j}-1\right) \frac{1}{m_{j}} \times \sum_{t=0}^{w}\left(\bar{\gamma}_{j}+\bar{\gamma}_{i}\right)^{-w+t} \\
\times{ }_{2} F_{1}\left(1, \alpha_{1}, \alpha_{2}, \alpha_{3}\right) \frac{(2 t-1) ! !}{2 t ! !} \cdot \frac{\pi(M-1) \cdot g_{p s k}^{-t}}{M}
\end{array}
$$

Where $w=m_{i}+m_{j}$

\subsection{M-QAM Modulation}

The average SER for M-QAM signals can be written using the same analysis as follow: 


$$
\begin{aligned}
P_{S E R}=\sum_{i=1}^{N} \prod_{\substack{j=1 \\
j \neq i}}^{N} P_{i} P_{j} & \times\left(\bar{\gamma}_{j}\right)^{m j} \Gamma\left(m_{i}+m_{j}-1\right) \frac{1}{m_{j}} \times \sum_{t=0}^{w}\left(\bar{\gamma}_{j}+\bar{\gamma}_{i}\right)^{-w+t} \\
& \times{ }_{2} F_{1}\left(1, \alpha_{1}, \alpha_{2}, \alpha_{3}\right) \frac{(2 t-1) ! !}{2 t ! !} \cdot \frac{\pi(M-1) \cdot g_{Q A M}^{-t}}{M}
\end{aligned}
$$

Where $g_{\mathrm{QAM}}=1.5 / M-1$.

These expressions can be used to evaluate SER for both M-PSK and M-QAM modulation schemes

\section{Numerical Results}

In this section, we present some numerical results to reveal the impact of different modulation schemes and channel affactive parameters on the average SER of rectangular of MPSK and M-QAM with arbitrarily ordered transmit antenna selection and receive MRC in Nakagami-m fading channels

Figure 2 shows the average SER with SNR for 4-PSK modulation signal using the Equation (17) for different values $m$ with $R=2$. We can see from the figure that $S E R$ decreases with the increment of $\mathrm{m}$, for example when the SNR $=20 \mathrm{~dB}$ the average SER equals 0.520468 , 0.445748 and 0.321638 for 4-PSK, when the $\mathrm{m}$ parameter $0.3,0.5$ and 1 respectively.

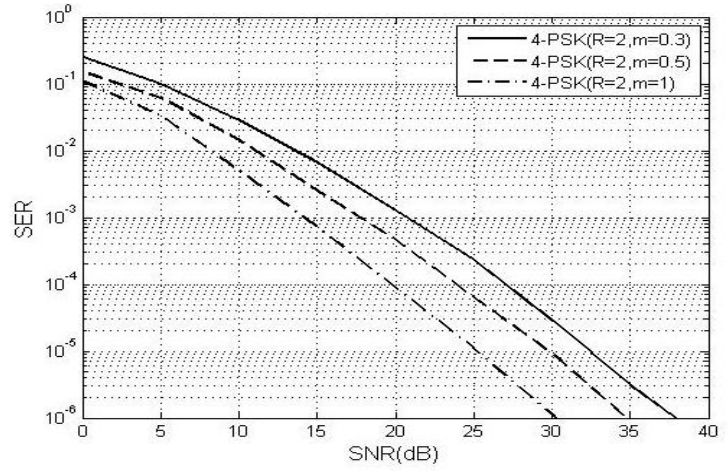

Figure 2. SER versus average SNR for 4-PSK modulation signals over Nakagami-m fading channel using MRC with $\mathrm{R}=2$ when $\mathrm{m}=0.3,0.5$ and1.

In Figure 3 we show the average SER for 4-QAM modulation signal using the equation (18) for different values $m$ with $R=2$. We can see from the figure that increasing the $m$ decreases the average SER, for example when the SNR $=20 \mathrm{~dB}$ the average SER equals $0.467837,0.354839$, and $1 \mathrm{e}-6$ for 4 -QAM, when the $\mathrm{m}$ parameter $0.3,0.5$ and 1 respectively.

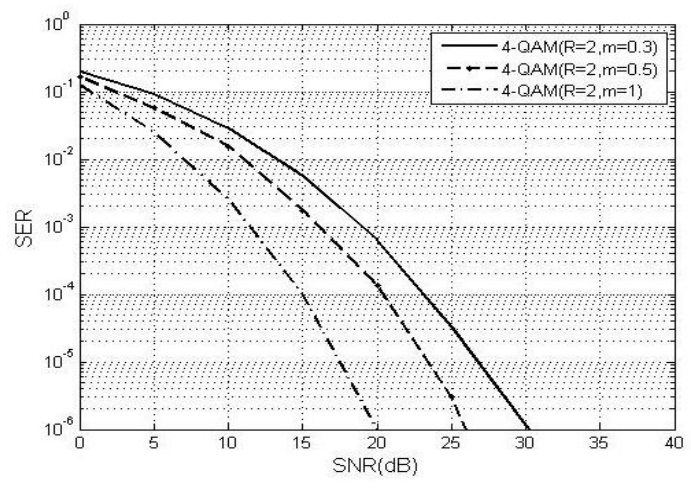

Figure 3. SER versus average SNR for 4-QAM modulation signals over Nakagami-m fading channel using MRC with $\mathrm{R}=2$ when $\mathrm{m}=0.3,0.5$ and1

TELKOMNIKA Vol. 16, No. 3, December 2015 : 546 - 552 
Figure 4 shows the average SER versus SNR of the cooperative system with M-PSK modulation signal, using the Equation (17) for different values R. And we use 4-PSK and 64PSK modulation signal, we can see that increasing the number of relays decreases the average SER.

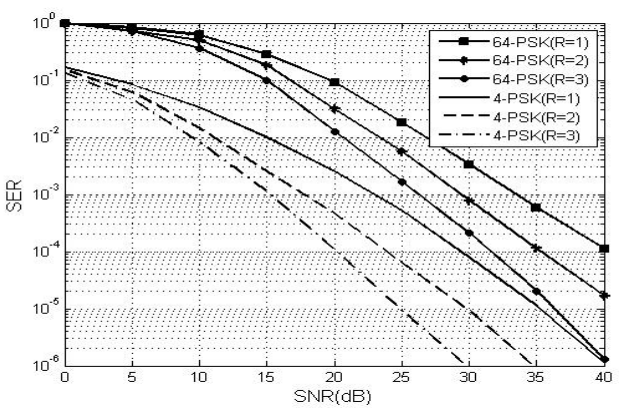

Figure 4. SER versus average SNR for 4-PSK and 64-PSK modulation signals over Nakagami-m fading channel using MRC with $\mathrm{R}=1,2$ and 3

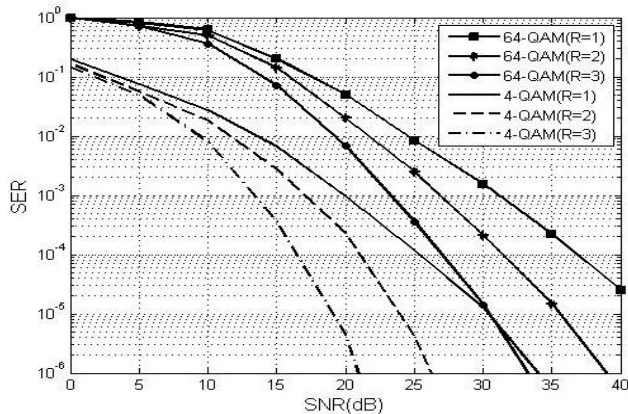

Figure 5. SER versus average SNR for 4-QAM and 64-QAM modulation signals over Nakagami-m fading channel using MRC with $\mathrm{R}=1,2$ and 3

Figure 5 shows the average SER versus SNR of the cooperative system with M-QAM modulation signal, using the Equation (18) for different values R. And we use 4-QAM and 64QAM modulation signal, we can see that increasing the number of relays decreases the average SER.

Figure 6 shows the comparison of the SER performance for the 4PSK, 64PSK, 4-QAM and 64-QAM modulation signals respectively with $R=2$ and $m=0.5$. As we see from the figure, if SNR $=20 \mathrm{~dB}$ the average SER equals 0.759331, 0.721408, 0.445748, 0.354839 for 64PSK, 64QAM, 4PSK and 4-QAM respectively. As it is clear from the figure, the 64PSK signal gets less SER as the average SNR increases for a constant number of relays.

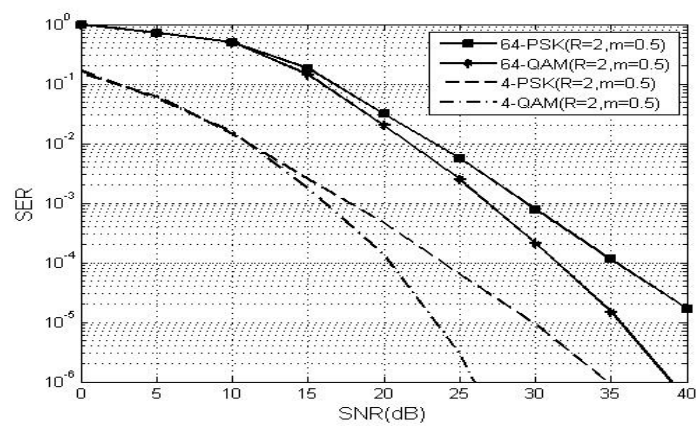

Figure 6. SER versus average SNR for 4-PSK , 64-PSK , 4-QAM and 64-QAM modulation signals over Nakagami-m fading channel using MRC when $\mathrm{m}=0.5$ and $\mathrm{R}=2$

\section{Conclusion}

In this paper, we investigate the variation of the number of relays and the $\mathrm{m}$ parameters on amplify-and-forward wireless cooperative network. We derive the PDF and the CDF from single half duplex channel with MRC. We considered the symbol error probability of AF Cooperative Networks with MRC and different modulation M-QAM and M-PSK in Nakagami-m fading with arbitrary $m \geq 0.5$. We derived an exact expression of MGF in term of the SNR. We further analyzed the error performance. From the simulation results, we obtain some guidelines to show the tradeoff between receiver complexity and SER performance under Nakagami-m fading conditions. 


\section{Acknowledgements}

This work was supported in part by the 863 project No.014AA01A701, 2015AA011303 and NSFC 61471408. Also by the National Natural Science Foundation of China (No. 60972016), Funds of Distinguished Young Scientists (No. 2009CDA150), ChinaFinish cooperation Project (No. 2010DFB10570) and two other projects (No. 2010ZX03003-00102 and No. 2012QN076).

\section{References}

[1] Zhu Y, Lin RD, Xu YY, Cai CX, Tang YC. Performance analysis of single relay selection in cooperative communication over Nakagami-m fading channels. Journal of Shanghai Jiaotong University (Science). 2013; 18(1): 25-28.

[2] Kong Hyung Yun, Vo Nguyen Quoc Bao. Capacity Analysis of Opportunistic Cooperative Networks under Adaptive Transmission over Rayleigh Fading Channels. Wireless Personal Communication. 2012; 62(2): 411-430.

[3] M Nakagami. The $m$-distribution, a general formula of intensity distribution of rapid fading. In Statistical Methods in Radio Wave Propagation. 1960: 3-36.

[4] H Mheidat, M Uysal. Impact of receive diversity on the performance of amplify-and-forward relaying under APS and IPS power constraints. IEEE Commun. Lett. 2006; 10(6): 468-470.

[5] Shin, Hyundong, Ju Bin Song. MRC analysis of cooperative diversity with fixed-gain relays in Nakagami-m fading channels. IEEE Trans. Wireless Commun. 2008; 7(6): 2069-2074.

[6] Kim YG, Beaulieu NC. New results on maximal ratio combining in Nakagami-m fading channels. IEEE International Conference (ICC). 2012: 4761-4765.

[7] VA Aalo, T Piboongungon, GP Efthymoglou. Another look at the performance of MRC schemes in Nakagami-m fading channels with arbitrary parameters. IEEE Trans. Commun. 2005; 53(12): 20022005.

[8] Z Chen, Z Chi, Y Liand B Vucetic. Error performance of maximal-ratio combining with transmits antenna selection in flat Nakagami-m fading channels. IEEE Trans. Wireless Commun. 2009; 8(1): 424-431.

[9] Z Chen, J Yuan, B Vucetic. Analysis of transmit antennaselection/maximal-ratio combining in Rayleigh fading channels. IEEE Trans .Vehicular Technology. 2005; 54(4): 1312-1321.

[10] Alam ASS, Goang Seog C. Performance of M-QAM, M-DPSK and M-PSK with MRC diversity in a Nakagami-m fading channel. Journal of Central South University. 2014; 21(4): 1347-1352.

[11] S Ikki, MH Ahmed. Performance analysis of cooperative diversity wireless networks over nakagami $\mathrm{m}$ fading channel. IEEE Commun. Letters. 2007; 11(4): 334-336.

[12] Mehemed A, Hamouda W. AF Cooperative CDMA Outage Probability Analysis in Nakagami- m Fading Channels. IEEE Trans .Vehicular Technology. 2013; 62(3): 1169-1176.

[13] IS Gradshteyn, IM Ryzhik. Table of Integrals, Series, and Products.7th ed. New York, USA: Academic Press. 2007.

[14] Ali A Mohammed, Li Yu, Manar Al-Kali. Performance Analysis of Amplify-and-Forward Cooperative Networks in Non-Identically Distributed Nakagami-m Channels with Best Relaying Selection. Journal of Communications. 2015; 10(6): 396-402. 